\title{
"The Calm before the Storm": Late Onset Glycine Encephalopathy Masquerading as Gastro-oesophageal Reflux
}

\author{
Shanawaz Hussain ${ }^{1}$, Manish Prasad ${ }^{2 *}$ and Santosh Mordekar ${ }^{1}$
}

${ }^{1}$ Department of Pediatric Neurology, Sheffield Children's Hospital, Sheffield, UK ${ }^{2}$ Department of Pediatric Neurology, Leicester General Infirmary, UK

\begin{abstract}
Glycine encephalopathy also known as 'Nonketotic Hyperglycinemia (NKH)' is an autosomal recessive disorder caused by a defect in the glycine cleavage enzyme complex (GCS). The classic neonatal form typically presents with generalised hypotonia, lethargy, seizures, apnoeas and usually death by one year of age. In this report, we describe an unusual presentation of an infantile onset glycine encephalopathy presenting at 8 weeks of age with symptoms suggestive of gastro-oesophageal reflux (GOR) episodes. A normal neonatal developmental course in the first few months of life is the exception in this phenotype and has been rarely described in the literature. To the best of our knowledge this is the first case of glycine encephalopathy reported with initial symptoms suggestive of GOR.
\end{abstract}

Keywords: Glycine encephalopathy; Gastro-oesophageal reflux; Sandifers; Dextromethorphan

\section{Introduction}

Non-ketotic hyperglycinaemia was first described by Gerritsen et al. in 1965 [1]. The defect in the glycine cleavage system results in an excessive accumulation of glycine in all tissues, but particularly in the CNS. Classification is based on the age at initial presentation and clinical outcome. This includes neonatal severe, neonatal mild, infantile mild, and infantile severe $[2,3]$.

In the classic neonatal form nearly two thirds of the children present within the first three days of life with progressive lethargy, muscular hypotonia, hiccups, myoclonic seizures, coma and ultimately death if not supported by ventilation $[2,4]$. Outcome for those who survive is poor with profound developmental delay, intractable seizures, spastic quadriparesis and microcephaly [3].

Nearly one fifth of the patients with GCE have a mild infantile form presenting in the first few months of life. Their outcome is much better and they learn to walk and acquire some motor skills $[3,4]$.

An elevated CSF/plasma glycine ratio suggests the diagnosis. Enzymatic confirmation can be done by measurement of glycine cleavage (GCS) enzyme activity in the liver. The 3 genes known to be associated with NKH are GLDC AMT and GCSH. Mutations associated with residual enzyme activity seem to be associated with a milder outcome and infantile presentation, and 2 mutations with no residual enzyme activity seem to be associated with severe outcome and neonatal onset [3-5].

The initial EEG typically shows a burst-suppression pattern that evolves into hypsarrhythmia or multifocal spike. MRI can be normal or show agenesis of the corpus callosum. Delayed myelination can be seen later in life. Agenesis of the corpus callosum is not specific and can be seen in various migrational and structural disorders of the CNS (e.g., Dandy-Walker malformation and lipoma of the interhemispheric fissure) [6]. Less common findings include retrocerebellar cysts with subsequent hydrocephalus [7]. A glycine peak on magnetic resonance spectroscopy is seen in the most severely affected infants and carries a poor prognosis.

To date, no effective treatment exists for this disorder. Therapy is focused on managing seizures by using sodium benzoate to reduce the plasma concentration of glycine. NMDA receptor antagonists (ketamine, dextromethorphan, felbamate, and topiramate) are also used in this condition [8].

\section{Case Report}

A 3 month old baby boy was referred with symptoms of poor feeding, sleepiness and abnormal jerky movements. He was born at term to healthy non-consanguineous parents and had normal initial development and developed a social smile at 6 weeks. He first developed significant back arching episodes at 8 weeks, thought to be related to gastro-oesophageal reflux for which he was treated with anti-reflux medications. The parents were also concerned that he was not fixing and following and had recently lost his social smile.

On admission he had a focal seizure with deviation of his eye and head towards the right side, he also appeared markedly encephalopathic. Initial examination showed him to be hypertonic with brisk reflexes throughout; he was not fixing or following. He had appropriate head growth along his centiles. An urgent EEG was done which was abnormal with a left sided temporal emphasis. He was started on vitamins and vigabatrin for his seizures. He continued to have seizures, managed with benzodiazepines and phenytoin.

In line with his early onset epileptic encephalopathy he had a full work up including LP, MRI, and ophthalmology assessment. His MRI was normal, but his CSF showed elevated glycine.

There was concern with regards to his CSF glycine which had come back high with a normal plasma glycine. For this reason a repeat LP was done which demonstrated a very high CSF and plasma glycine with a high ratio: diagnostic of non-ketotic Hyperglycinaemia. The diagnosis and implications as well as prognosis were discussed with

*Corresponding author: Dr. Manish Prasad, Department of Paediatric Neurology Leicester Royal Infirmary, Infirmary Square, Leicester LE1 5WW, UK, E-mail: m.prasad@nhs.net

Received February 20, 2013; Accepted February 27, 2013; Published March 01 2013

Citation: Hussain S, Prasad M, Mordekar S (2013) "The Calm before the Storm": Late Onset Glycine Encephalopathy Masquerading as Gastro-oesophageal Reflux.

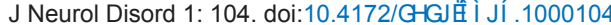

Copyright: (C) 2013 Hussain S, et al. This is an open-access article distributed under the terms of the Creative Commons Attribution License, which permits unrestricted use, distribution, and reproduction in any medium, provided the original author and source are credited. 
Citation: Hussain S, Prasad M, Mordekar S (2013) "The Calm before the Storm": Late Onset Glycine Encephalopathy Masquerading as Gastro-

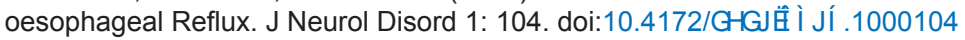

Page 2 of 2

\section{What is already known on this topic?}

- GCE can present in three forms with the most commonest presenting within the first few days of life.

- The prognosis remains bleak with death usually occurring within the first year of life.

What this case report adds?

- The widely used pediatric mantra "all that vomits, is not reflux!" holds true and has a wide differential diagnosis.

- To consider non-ketotic hyperglycinemia in infants presenting with Sandifer's syndrome.

- NMDA receptor agonists showed a significant improvement in encehaplopathy and seizures in our patient and may have a role in the management of GCE.

Table 1: Summary of what is known on this topic and what this case report adds.

parents. A liver biopsy confirmed the diagnosis. A metabolic consult was made and he was started on oral dextromethorphan and a ketogenic diet. There was marked improvement in encephalopathy and his seizures in the first week of commencing the dextromethorphan and the ketogenic diet.

\section{Discussion}

Several forms of atypical GCE have been described. Broadly based on the age of initial presentation they can be divided into 3 main types: Neonatal (0-4 weeks), Infantile (5 weeks to 2 years) and lateonset (beyond 2 years) [8].

In general, the presentation in the atypical neonatal form is similar to the classic neonatal form with hypotonia and apnoeic episodes but the outcome is significantly better [7] and rarely completely normal development has been reported [8].

We believe the initial presentation in our index case may be more in keeping with the classic neonatal form with history of excessive sleepiness and unarousable (encephalopathic) for the first 2-3 days of life as noticed both by parents and midwives. Also increased jerky movements (possible hiccups) experienced by his mother in the last 3-4 weeks before delivery is only described in the classic neonatal forms. However he didn't follow any known usual course and in fact improved dramatically and also achieved normal developmental milestones in the form of social smiling at around 6 weeks of age. We understand that this is subjective and a lot of normal babies may be unusually sleepy for the first few days of life. However retrospectively mother is very certain about the hiccup like jerky movements she felt in the later part of her pregnancy and comparing with her elder son, he was unusually sleepy for the first few days.

Although there have been few case reports of transient GCE [9], their course is very different with acute presentation in neonatal age group followed by normal or near normal development subsequently with no secondary deterioration of the kind described in our index case.

The most common presentation of the atypical GCE is the infantile form. Typically infants with this form have nearly normal development for the first 6 months of life and then present with mild to moderate developmental delay. Seizures, occurring in less than half of the patients, can be of any type (epileptic spasms, complex partial, tonic or generalised tonic-clonic) and are relatively easy to control [8].

\section{Conclusion}

Our index case developed normally until 8 weeks of age and also achieved a social smile from 6 weeks of age. His presentation at 8 weeks of age masquerading as gastro-oesophageal reflux is very unusual and is not described in the literature. His deterioration was fairly rapid which again generally is not the expected course for an infantile onset GCE, although occasionally reported $[2,10]$. Their outcome is generally as poor as the classic neonatal form with profound developmental delay, intractable seizures and spasticity [3] (Table 1).

\section{Acknowledgments}

The authors wish to acknowledge the help of the metabolic department and also the neurophysiology department for their assistance with this case.

\section{Author Contributions}

Shanawaz Hussain acquired the information and authored the first draft of the manuscript. Manish Prasad helped with revision and the final draft of the manuscript. Santosh Mordekar gave guidance and critical review on the manuscript.

\section{Declaration of Conflicting Interests}

The authors declare no potential conflicts of interest with the research, authorship and/or publications of this article.

\section{Funding}

The authors received no financial support for the research, authorship and/ or publication of this article.

\section{References}

1. Gerritsen T, Kaveggia E, Waisman HA (1965) A new type of idiopathic hyperglycinemia with hypo-oxaluria. Pediatrics 36: 882-891.

2. Hennermann JB, Berger JM, van Hove JLK (2006) Prognostic factors indicating the outcome of glycine encephalopathy: a follow-up of 40 children. Journal of Inherited Metabolic Disease 29: 49.

3. Hennermann JB (2006) Clinical variability in glycine encephalopathy. Future Neurology 1: 621-630.

4. Hoover-Fong JE, Shah S, Van Hove JL, Applegarth D, Toone J, et al. (2004) Natural history of nonketotic hyperglycinemia in 65 patients. Neurology 63 : 1847-1853.

5. Kure S, Tada K, Narisawa K (1997) Nonketotic hyperglycinemia: biochemical molecular, and neurological aspects. Jpn J Hum Genet 42: 13-22.

6. Sakata Y, Owada Y, Sato K, Kojima K, Hisanaga K, et al. (2001) Structure and expression of the glycine cleavage system in rat central nervous system. Brain Res Mol Brain Res 94: 119-130.

7. Dinopoulos A, Matsubara Y, Kure S (2005) Atypical variants of nonketotic hyperglycinemia. Mol Genet Metab 86: 61-69.

8. Korman SH, Boneh A, Ichinohe A, Kojima K, Sato K, et al. (2004) Persistent $\mathrm{NKH}$ with transient or absent symptoms and a homozygous GLDC mutation. Ann Neurol 56: 139-143.

9. Zammarchi E, Donati MA, Ciani F (1995) Transient neonatal nonketotic hyperglycinemia: a 13-year follow-up. Neuropediatrics 26: 328-330.

10. Van Hove JLK, Mahieu V, Schollen E (2003) Prognosis in nonketotic hyperglycinemia. J Inherit Metab Dis 26: S71. 